\title{
Determination of the permittivity of soils by use of double transmission measurements
}

\author{
B. Will and M. Gerding \\ Ruhr-Universität Bochum, Institute of High Frequency Engineering, Universitätsstr. 150, 44801 Bochum, Germany
}

\begin{abstract}
Delay time measurements, e.g. time domain reflectometry (TDR), are a well-established method for the measurement of permittivity of various materials, especially soils. However, common measurement systems only provide one average value of the dielectric constant along the length of the TDR probe. This contribution deals with an advanced application of the TDR principle, the so-called double transmission method, for the determination of the water content of soil along a probe. To apply the advanced TDR technique, a probe, realized by a combination of a transmission line and a dielectric obstacle, which can mechanically be moved along the probe, is used. The probe is inserted into the soil to measure the effective soil permittivity. Thus, the water content along the probe can be estimated by means of the effective permittivity. Based on the known mechanical position of the reflection at the end of the probe and the position of the obstacle, the measured delay time can be used as a measure for the effective dielectric constant of the environment surrounding the obstacle. Thus, it is possible to determine the effective dielectric constant with a spatial resolution given by the step size of the obstacle displacement.
\end{abstract}

\section{Introduction}

Several well-established techniques have been developed for the determination of the permittivity in homogenous and inhomogeneous materials (Robinson et al., 2003). They all have in common, that their measurement results are limited to the mean value of the permittivity of the respective material of interest.

Due to the existing relation between the permittivity and the moisture of a material probe, permittivity measurements became a well established method for the determination of the water content of soils (Kupfer, 2005; Kupfer et al., 2000;

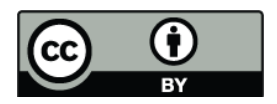

Correspondence to: $\mathrm{B}$. Will (bianca.will@rub.de)
Hoekstra and Delaney, 1974). By the use of capacitive sensors or time delay measurements it is possible to determine the mean value of the water content along a sensor which is penetrating into the medium.

This contribution deals with an improved measurement method, capable to determine the distribution of the permittivity of soils with an adjustable spatial resolution along a sensor, which is penetrating into the medium. By this, the method may be a solution for the upcoming needs to determine the moisture distribution inside soils for observing e.g. the infiltration of moisture on waste dumps and dikes and for solving the increasing numbers of industrial applications like the characterization of emulsions.

The conventional TDR (Time Domain Reflectometry) technique serves as the basis for the presented measurement principle, which itself can be subdivided into three alternative solutions: the reflection measurement, the transmission measurement and the double transmission measurement. All solutions make use of the delay time measurement of the transmitted or reflected signal, which is correlated to the permittivity of the material surrounding the sensor. The main difference between the three alternative solutions are the configurations of the measurement ports and the implementation of the local interaction between the electromagnetic signal travelling along the probe and the surrounding material. The following considerations focus on the double transmission concept, which is expected to be the most robust and reliable concept of the three mentioned alternatives.

\section{Basics of the double transmission measurement}

The basic functional principle of the double transmission measurement system is illustrated in Fig. 1. Figure 1a illustrates the setup for the reference measurement, while b, c, d illustrate an arbitrary measurement situation to determine the permittivity of the material located around the probe. The measurement setup itself mainly consists of the following components: 


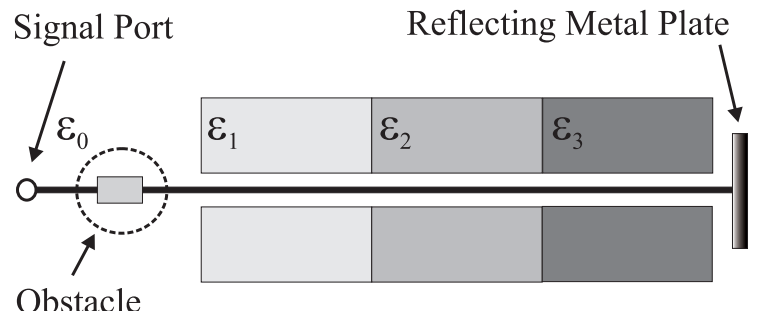

a)

Obstacle

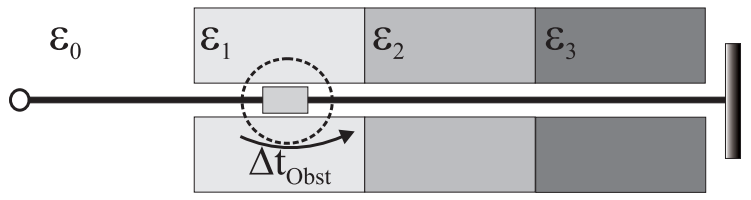

b)

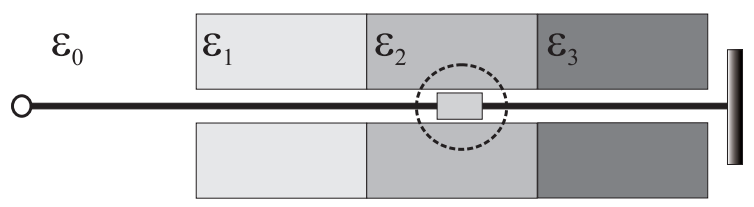

c)

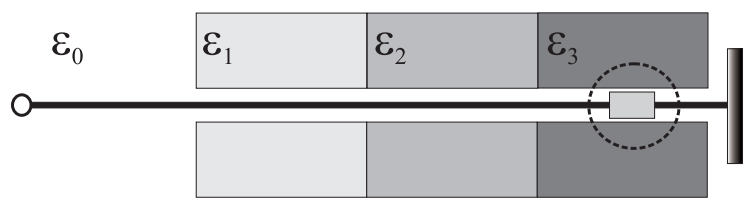

Fig. 1. Functional principle of the double transmission measurement: (a) Obstacle in reference position; (b), (c), (d) Obstacle in different measurement positions.

- A conductive single rod or cable, acting as a transmission line.

- The so called "obstacle", a dielectric cylinder, which can mechanically be moved along the waveguide, to provide a movable local electromagnetic disturbance.

- A metal plate at the end of the electromagnetic waveguide, reflecting the electromagnetic wave

The transmission line and the obstacle are covered by a thinwalled plastic tube for practical reasons. By this, the complete probe can directly be inserted into the medium while the space for the obstacle movement is still guaranteed by the plastic tube. Finally, the complete setup is connected to the measurement electronic via one signal port at the beginning of the probe. Thus, the signal flow is basically compatible with a conventional industrial baseband TDR-System.

Generally, the measurement principle is based on the propagation delay time of a transmitted electromagnetic pulse. The signal propagates along the probe, guided by the single rod inside the plastic tube. Excluding the volume in the vicinity of the obstacle, the electromagnetic field is concentrically concentrated around the transmission line with hardly any field components outside the plastic tube, as it is illustrated in Fig. 2a and c. At the end of the probe, the signal is reflected
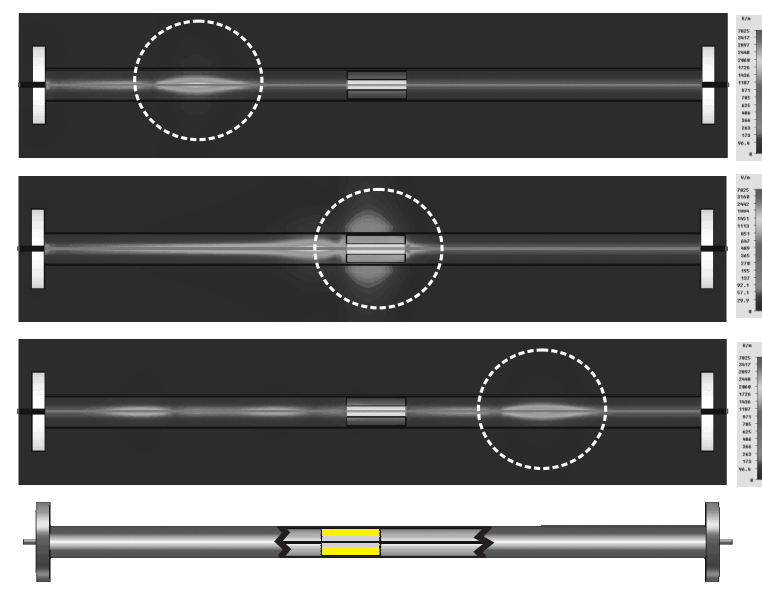

c)

Fig. 2. EM-simulation illustrating the movement of the electromagnetic pulse along the probe: (a), (c) The EM-field is concentrically concentrated around the waveguide; (b) The EM-field penetrates into the surrounding of the probe.

by the metallic plate and travels back in the opposite direction. Following the signal path, it is obvious that the signal is transmitted twice through the region of interest, illustrating the name of the introduced "Double Transmission" principle. At the obstacle, the electromagnetic field penetrates into the surrounding of the probe and thus penetrating into the material, which has to be characterized (Figs. 1b, 2b). This displacement of the electromagnetic field and the penetration of the outer material increases the propagation delay by $\Delta t_{\text {obst }}$ and gives a measure of its permittivity. By mechanically moving the obstacle along the probe it is possible to characterize the region of interest at nearly any arbitrary position, as illustrated in Fig. 1b, c, d.

The displacement of the electromagnetic field into the surrounding, caused by the obstacle, has been validated via simulations and is due to the difference between the permittivity inside the plastic tube, which is close to the permittivity in free-space and the comparable high permittivity of the dielectric obstacle. The dielectric obstacle itself consists of a ceramic material $\left(\varepsilon_{r} \approx 15\right)$. As a matter of fact, the electromagnetic wave takes the way providing the highest possible propagation speed. Estimating a permittivity of the area surrounding the obstacle of less than $\varepsilon_{r} \approx 15$, this forces the electromagnetic field to penetrate the outer medium, following the relation $c=c_{0} / \sqrt{\varepsilon_{r_{\mathrm{eff}}}}$.

Thus, the propagation speed and the corresponding signal delay time of the transmitted signal is a measure for the relative effective permittivity of the surrounding material.

Initially, the obstacle is placed in its reference position outside the material under test, as illustrated in Fig. 1a, in order to achieve the overall signal delay time $\Delta t_{\text {ref }}$ as a reference value:

$\Delta t_{\text {ref }}=\Delta \tilde{t}_{\text {obst }}+\Delta t_{\text {probe }}$ 


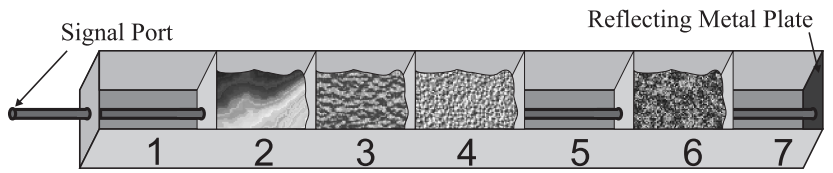

Fig. 3. Simplified schematic overview of the double transmission measurement setup which is used for the exemplary measurement results.

$\Delta \tilde{t}_{\text {obst }}$ represents the delay time, caused by the obstacle in its reference position and $\Delta t_{\text {probe }}$ implies the signal delay time along the rest of the probe. The reference position of the obstacle is nearly arbitrary, as long as the obstacle is located outside the material under test.

In general, the delay time caused by the obstacle can be described as follows:

$\Delta t_{\mathrm{obst}}=2 \cdot l_{\mathrm{obst}} \frac{\sqrt{\varepsilon_{r_{\mathrm{eff}}}}}{c_{0}}$

The effective permittivity $\varepsilon_{r_{\text {eff }}}$ mainly comprises the permittivity of the plastic tube and of the outer material, which is penetrated by the electromagnetic field. The permittivity of the obstacle itself has nearly no influence on the effective permittivity $\varepsilon_{r_{\text {eff }}}$, as it has been verified by simulations, due to the effect, that the electromagnetic field is mainly not penetrating into the obstacle. In fact, the electromagnetic field is mainly pushed into the surrounding of the obstacle. During the reference measurement, the obstacle should preferably be covered by air, so that $\varepsilon_{r_{\text {eff }}}$ is mainly defined by the permittivity of air $\left(\varepsilon_{r} \approx 1\right)$. Thus the effective permittivity $\tilde{\varepsilon}_{r_{\text {eff }}}$ in Eq. (2) of the active surrounding material during the reference measurement can be approximated to be close to 1 , leading to:

$$
\begin{aligned}
\Delta \tilde{t}_{\mathrm{obst}} & =2 \cdot l_{\mathrm{obst}} \frac{\sqrt{\tilde{\varepsilon}_{r_{\mathrm{eff}}}}}{c_{0}} \\
& \approx 2 \cdot l_{\mathrm{obst}} \frac{1}{c_{0}}
\end{aligned}
$$

$l_{\text {obst }}$ is the mechanical length of the obstacle. Due to the double transmission principle, the transmitted signal passes the obstacle twice, which is taken into account by the factor of 2 in Eq. (3). $\Delta t_{\text {probe }}$ describes the signal delay time, caused by the rest of the probe, excluding the obstacle. In a first approximation $\Delta t_{\text {probe }}$ can be assumed to be independent of the obstacle position. This leads to the following equation for an arbitrary obstacle position (index $i$ ) as illustrated in Fig. 1b, $\mathrm{c}, \mathrm{d}$ :

$\Delta t(i)=\Delta t_{\mathrm{obst}}(i)+\Delta t_{\text {probe }}$

Defining the difference $\Delta T(i)$ between $\Delta t(i)$ and $\Delta t_{\text {ref }}$ to be a measure for the effective permittivity of the material at the obstacles position, this yield:

$\Delta T(i)=\Delta t(i)-\Delta t_{\mathrm{ref}}$
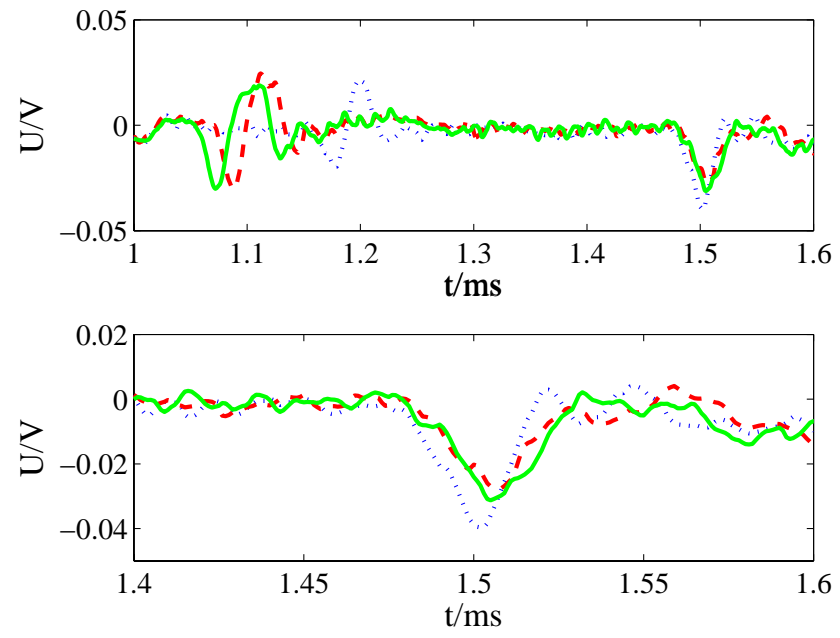

Fig. 4. Qualitative illustration of the different propagation delay times, caused by different materials under test aligned at three different positions along the probe.

$$
\begin{aligned}
& =\Delta t_{\mathrm{obst}}(i)-\Delta \tilde{t}_{\mathrm{obst}} \\
& =2 \cdot \frac{l_{\mathrm{obst}}}{c_{0}}\left(\sqrt{\varepsilon_{r_{\mathrm{eff}}(i)}}-\sqrt{\tilde{\varepsilon}_{r_{\mathrm{eff}}}}\right)
\end{aligned}
$$

Simplifying the equation by the use of the relation from Eq. (3) results in:

$\varepsilon_{r_{\mathrm{eff}}}(i)=\left(\frac{1}{2} \frac{\Delta T(i) c_{0}}{l_{\mathrm{obst}}}+1\right)^{2}$

Thus, it is possible to determine the effective permittivity $\varepsilon_{r_{\text {eff }}}(i)$ in the immediate vicinity of each arbitrary obstacle position. This yields a measurement system for the determination of dielectric profiles along the probe, e.g. by moving the obstacle stepwise along known positions. Furthermore it should be remarked, that the clearly layered measurement scenarios in Fig. 1 are simplified examples, only. The functionality of the introduced double transmission principle exceeds these examples and is also capable of handling mixed layers and irregular layered materials.

\section{Measurements}

The capability of the double transmission principle is verified by the following exemplary measurement results: the basis for these measurement is a test fixture, similar to the illustration in Fig. 3. According to the most probable application, the probe will be used in a vertical position, but for practical reasons, the test fixture is mounted in a horizontal position. This has no negative effect on the measurement principle and furthermore allows an easy placement of well defined test scenarios. The probe itself is fixed in the center of a horizontally placed box, which acts as a container for the material under test. The box itself is subdivided into single 


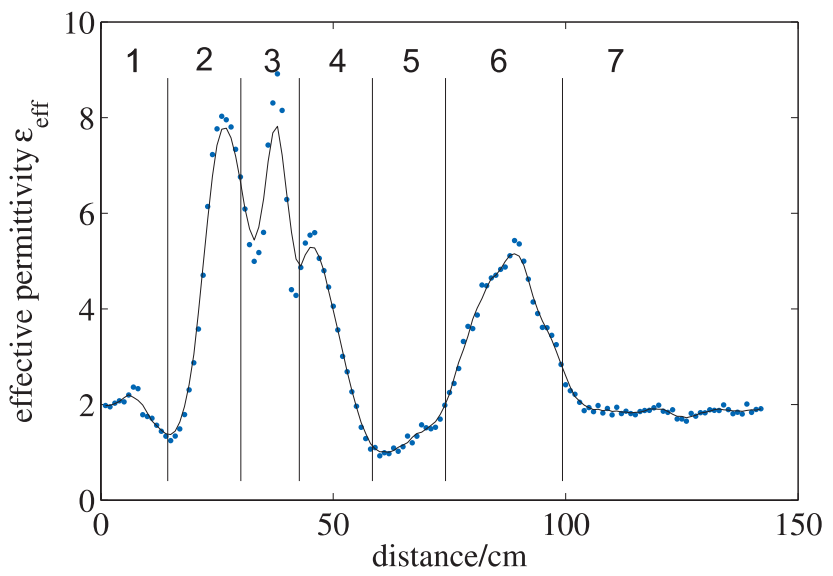

Fig. 5. Calculated effective permittivity based on measurement results corresponding to the measurement setup, illustrated in Fig. 3. (The step width of the obstacle movement is $1 \mathrm{~cm}$. The solid line is the 5-point moving average curve of all single datapoints (dotted line).)

cavities, allowing the alignment of different "discrete" material layers for testing purposes. The signal port is located on the left side of the test fixture and the reflecting metal plate is located at the right side of the test fixture, directly at the end of the probe. According to the exemplary measurement scenario, the illustration in Fig. 3 shows four of the seven tests volumes filled with sand, each having a different humidity.

The propagation delay time of the transmitted signal measured at each obstacle position is a measure for the effective permittivity of the material along the probe position. The effect of the surrounding of the obstacle on the propagation delay time is qualitatively shown in Fig. 4. The curves show the resulting time-domain signals for arbitrary obstacle positions. The pulse signals around the particular time of $1.5 \mathrm{~ms}$ show the resulting time delay, which is used for calculating the effective permittivity by the use of Eq. (6).

In order to determine a permittivity profile, the obstacle stepwise has to be moved along the probe. The step width is a user-defined parameter and has a direct influence on the spatial resolution of the final permittivity profile. Here the step width is chosen to be $1 \mathrm{~cm}$. Moving the obstacle along the complete probe and characterizing the test fixture as it is illustrated in Fig. 3 yields the permittivity profile in Fig. 5. As it can clearly be seen, the determined effective permittivity of the surrounding of the probe is always greater than 1 , even if the test volume in three of the seven cavities is air. This is due to the mechanical design of the probe and has to be taken into account when finally calculating the absolute permittivity of the surrounding material under test.

Even if the test fixture gives the impression, that the presented principle is limited to well layered materials only, it has to be remarked that especially the double transmission method can handle smooth and homogeneous material mix- tures, because the principle does not rely on the reflection of the separation layer between two materials, as it is the case by already known measuring principles (Huebner et al., 2007; Kupfer et al., 2007; Scheuermann et al., 2007). In case of the double transmission principle, the reflection of the separation layer and the reflection of the obstacle itself could be used to have some additional information to increase the reliability and the robustness of the system, but so far it has not been taken into account.

\section{Conclusion}

The so-called double transmission principle is introduced as an advanced and advantageous technique for the determination of the water content of soil along a probe. The basis of the advanced TDR technique is a probe, realized by a combination of a waveguide and a dielectric obstacle, which can mechanically be moved along the probe acting as the key component for the allocation between the measuring value and the place where it is taken. This leads to the system capability of characterizing permittivity profiles with an arbitrary step size. The step size is directly related to the resulting spatial resolution of the profile and can be adapted problem specific to the individual needs. Furthermore the system electronic is based on the well known TDR technique and can easily be adapted to existing industrial TDR-measurement systems as they are used in level measurements, for example.

\section{References}

Hoekstra, P. and Delaney, A.: Dielectric properties of soils at UHF and microwave frequencies, J. Geophys. Res, 79, 1699-1708, 1974.

Huebner, C., Schlaeger, S., and Kupfer, K.: Spatial Water Content Measurement with Time-Domain Reflectometry, tm-Tech. Mess., 74, 316-326, 2007.

Kupfer, K.: Electromagnetic Aquametry: Electromagnetic Wave Interaction With Water And Moist Substances, Springer, 2005.

Kupfer, K., Kraszewski, A., and Knöchel, R.: RF and Microwave Sensing of Moist Materials, Food and Other Dielectrics, WileyVCH, 2000.

Kupfer, K., Trinks, E., Schafer, T., Wagner, N., and Hubner, C.: Determination of Moisture and Density Distributions using TDRSensors, tm-Tech. Mess., 74, 298-307, 2007.

Robinson, D., Jones, S., Wraith, J., Or, D., and Friedman, S.: A Review of Advances in Dielectric and Electrical Conductivity Measurement in Soils Using Time Domain Reflectometry, Vadose Zone J., 2, 444-475, 2003.

Scheuermann, A., Bieberstein, A., Schlaeger, S., and Becker, R.: Optimized Sensor Design for the Determination of the Spatial Moisture Distribution in Electrical Lossy Soils, tm-Tech. Mess., 74, 308-315, 2007. 